\title{
A Rough Calculation relating the extremely small Cosmological Constant To the extremely large QFT Vacuum Energy Density
}

\author{
Remi Cornwall \\ University of London Alumni Society, Malet Street, London WC1E 7HU \\ http://webspace.qmul.ac.uk/rocornwall http://ulondon.academia.edu/RemiCornwall
}

\begin{abstract}
This enquiry follows the investigation into a propulsion system purportedly utilising the QED vacuum as reactive momenergy. The QFT vacuum is contentious because the "naïve" value for it is extraordinarily large, yet on the cosmic scale it is hardly present. This begs the question as to whether it is really real and further highlights the problem between General Relativity on very large scales, with Quantum Mechanics on very small scales. We find a mathematical procedure that: to the $1^{\text {st }}$ order removes the "embarrassing" QFT vacuum constant from the Einstein tensor and then covers nearly all of the 120 orders of magnitude difference between the Cosmological Constant and Vacuum Energy by introducing it as an higher order correction in $\left(\mathrm{G} / \mathrm{c}^{4}\right)^{3}$. There is a proviso for further work, that the difference of a few orders we calculate, might be made up by considering fluctuations, running constants in the QFT vacuum, extra degrees of freedom/dimensions, Inflation or even non-constant G.
\end{abstract}

Keywords: Cosmological constant, QFT vacuum, Perturbation expansion, Einstein tensor

\section{Introduction}

The author is working on the theoretical underpinnings of a putative electromagnetic propulsion device[1-2] that pushes against the ground state of the vacuum. It is imperative to establish in the light of observations, both cosmological and small-scale on the field ground state, whether it exists as required for comparatively macroscopic engineering science use or whether it is too small.

The "zeropoint" is contentious[3]; it should exist as many phenomena are attributed to it: Lamb shift, hyperfine splitting of spectral lines, van der Waal/London/Casimir forces and even the liquid state of helium near absolute zero[4-5]. However when the techniques of analysis used for the quantum harmonic oscillator is applied to the field, an extraordinarily large vacuum constant arises[6]. According to General Relativity this vacuum energy should be present in the stress-energy tensor $\mathrm{T}_{00}$ component and lead to rapid universal collapse and not the expansion. However we are seeing accelerating expansion, with an extremely small cosmological constant and negative pressure would account for this[7]. It is reasonable to model this effect as an ideal liquid in the following stressenergy tensor[6,8],

$$
T_{\mu v}=(\rho+p) u_{\mu} u_{v}+p g_{\mu v}
$$

And an homogeneity constraint (Lorentz invariance) renders the first term zero such that (signature -1,1,1,1),

$$
T_{\mu v_{v a c}}=-\rho_{v a c . \operatorname{Cosmo}} g_{\mu v}
$$

Whereupon the Einstein Field Equations become,

$$
\begin{aligned}
& R_{\mu v}-\frac{1}{2} R g_{\mu v}=\frac{8 \pi G}{c^{4}}\left(T_{\mu v_{\text {mass }, \text { energ }}}+T_{\mu v_{v a c}}\right) \\
& \Rightarrow E_{\mu v}+\Lambda g_{\mu v}=\kappa T_{\mu v_{\text {mass,energy }}}
\end{aligned}
$$

Where we have replaced the Ricci tensor and scalar curvature with the Einstein tensor thus,

$$
E_{\mu v}=R_{\mu v}-\frac{1}{2} R g_{\mu \nu}
$$

And replaced the constant of proportionality on the RHS,

$$
\kappa=\frac{8 \pi G}{c^{4}}
$$

And moved the vacuum energy tensor to the LHS with the constant,

$$
\Lambda=\frac{8 \pi G}{c^{4}} \rho_{v a c . \text { Cosmo }}
$$

Astronomical observations give a figure for $\rho_{\text {vac.Cosmo }}$ of about $10^{-9} \mathrm{~J} / \mathrm{m}^{3}$ (as well as universal expansion, see first note in [7]) which corresponds to $\Lambda^{-1 / 2}$ of about $10^{9}$ light-years.

The vacuum state for all types of field, fermion and boson is modelled as an harmonic oscillator, for which an estimation of the energy density of the vacuum state follows this procedure[6]:

$$
\rho_{\text {vac.QFT }}(\omega)=\frac{2}{8 \pi^{3}} \int \frac{1}{2} \hbar \omega d^{3} k=\left[\frac{\hbar \omega^{3}}{2 \pi^{3} c^{3}}\right]_{0^{+}}^{\omega_{\text {Planck }}} \text { eqn. } 7
$$


This is calculated to be some $10^{111} \mathrm{~J} / \mathrm{m}^{3}$, leaving 120 orders of magnitude difference between $\rho_{\text {vac.QFT }}$ and $\rho_{\text {vac.Cosmo }}$. We seek to ask if there is a relation between these two quantities and establish a much nearer relationship between them. Hopefully too we will have a mathematical procedure to contend with normal ordering [5] (perhaps be more physically justified than) or just removing it.

\section{The mathematical procedure}

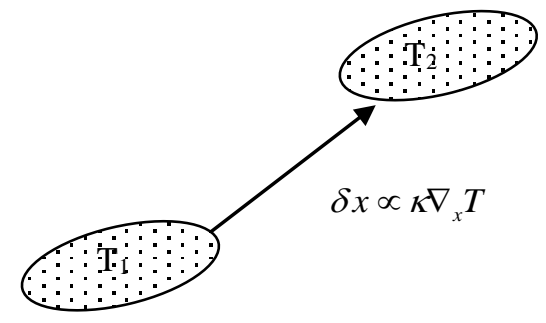

Figure 1

Let us consider a region (figure 1) where the stressenergy tensor is at one value $\left(\mathrm{T}_{1}\right)$ and then at another $\left(\mathrm{T}_{2}\right)$ a small distance away $(\delta \mathrm{x})$. The stressenergy, itself, is curving space and we shall make this distance proportional to the covariant derivative of the Einstein tensor. We shall use a covariant Taylor expansion of the Einstein-Hilbert Field Equation with the cosmological constant, $\kappa$, on the RHS so if,

And

$$
E=\kappa\left(T_{\text {mass,energy }}+T_{\text {vac }}\right)
$$

$$
\delta s=\sqrt{g^{\mu v} d x_{\mu} d x_{v}} \quad \text { eqn. } 8
$$

Now,

$$
\delta x=f(\delta s)
$$

And

$$
\begin{aligned}
& \delta x \propto \nabla_{x} E \\
& \Rightarrow \delta x \propto \kappa \nabla_{x} T
\end{aligned}
$$

Then,

$$
\begin{aligned}
& E_{\mu v}(x+\delta x) \\
& \square E_{\mu \nu}(x)+\nabla_{x} E_{\mu \nu}(x)\left(\delta \kappa \nabla_{x} T\right) \\
& \quad+\frac{1}{2 !} \nabla_{x}^{2} E_{\mu \nu}(x)\left(\delta \kappa \nabla_{x} T\right)^{2}+\ldots
\end{aligned}
$$

This has a dual purpose of considering points near to our 4-vector position and also viewing it as a perturbation expansion.

\subsection{Removing the QFT vacuum to $1^{\text {st }}$ order in Einstein tensor}

If we consider region 1 (figure 1) as the classical vacuum (without consideration of the Cosmological constant, nor the QFT vacuum), then the stress-energy is exactly zero. We can then consider the second region (figure 1) as containing any field or mass-energy that we conventionally put into the stress-energy. Moving from region 1 to 2 integrates the $2^{\text {nd }}$ term (figure 1 ). The $2^{\text {nd }}$ term is also a statement of the conservation of momentum,

$$
\nabla_{\mu} T_{v}^{\mu}=0
$$

Technically, since $\nabla_{\mu} T_{v a c . Q F T}=0$ we can leave the embarrassing figure out of the tensor anyway, along the lines of putting in (or not) an integration constant; if we don't observe its effects as expected, we can leave it out or put it in at higher order.

\subsection{The QFT vacuum enters at $2^{\text {nd }}$ order in Einstein tensor}

We shall now consider empty space and the Cosmological constant. Using the same trick, we can make region 1 in figure 1 the classical vacuum (although it couldn't exist without the QFT vacuum) and region 2 the QFT vacuum and write by eqn. 11 ,

$$
\begin{aligned}
& E_{\mu \nu}(x+\delta x) \square \nabla_{x}^{2} E_{\mu \nu}(x)\left(\delta \kappa \nabla_{x} T\right)^{2} \\
& \Rightarrow \\
& E_{\mu \nu}(x+\delta x) \square \nabla_{x}^{2} E_{\mu \nu}(x)\left(\delta \kappa \nabla_{x} T\right)^{3}
\end{aligned}
$$

(The factor $1 / 2$ ! in eqn. 11 is neither here nor there in this rough calculation).

Trying simplest cases first and knowing that on small scales that the Cosmological constant hardly curves space, we can side-step the complicated machinery of the covariant derivative, note the result of section 2.1 and eqn. 2 , then calculate the integral of the divergence of $\mathrm{T}_{00}$ over an infinitesimal volume of the line element eqn. 8,

$$
\int_{V} \nabla T_{00} d V=\iint_{S} T_{00} \cdot 4 \pi g^{\mu v} d x_{\mu} d x_{v} \quad \text { eqn. } 14
$$

This implies eqn. 2 but multiplied by $\kappa^{3}$ (eqn. 13), so we can write,

$$
\begin{aligned}
& E=\kappa T_{\text {mass,energy }}+\kappa^{3} g_{\mu v} T_{\text {vac.QFT }} \\
& \Rightarrow E \rightarrow \kappa T_{\text {mass,energy }}+g_{\mu \nu} T_{\text {vac.Cosmo }} \\
& \Rightarrow E_{\mu \nu}+\Lambda g_{\mu v}=\kappa T_{\mu v_{\text {mass,energy }}}
\end{aligned}
$$

The factor $\kappa^{3} \square 10^{-129}$ (eqn. 5) multiplying $T_{\text {vac.QFT }}$ brings it closer to the observed $\rho_{\text {vac.Cosmo }}$, save perhaps some fluctuation/running constant/inflation effect $[6,10]$ in $\rho_{\text {vac.QFT }}$ at small scales, which would then make up the $10^{9}$ magnitude difference; 
this is just a factor of a thousand for each axis $\mathrm{x}, \mathrm{y}, \mathrm{z}$. We've also dropped several constants in our rough calculation taking $10^{2}$ or so out. This is definitely getting into the ballpark, though.

\section{Conclusion}

A putative perturbative expansion of the Einstein tensor has be lain out that cancels the massive vacuum energy to $1^{\text {st }}$ order by an acceptable mathematical sleight of hand and introduces it at the second order in the said tensor, which corresponds to third order in $\mathrm{G} / \mathrm{c}^{4}$ of the stress energy tensor. This has brought the discrepancy between the observed astronomical vacuum energy and to what quantum field theories predict the vacuum energy to be to within a few orders of magnitude. Further work looking at fluctuations, running constants, extra degrees of freedom/dimensions, inflation theories or even $\mathrm{G}$ varying and becoming stronger on very small scales may bring it closer still.

\section{$\underline{\text { References }}$}

1. Cornwall, R.O., A Mechanism for Propulsion without the Reactive Ejection of Matter or Energy. Preprints, 2019 (https://www.academia.edu/38062548/A Mechanism for_Propulsion_without The Reactive_Ejection_of_Matter_or_Energy ).

2. Graham Lahoz, Observation of static electromagnetic angular momentum in vacuo. Nature, 1980. 285(154).

3. Saunders, S. Is the Zero-Point Energy Real? Ontological Aspects of Quantum Field Theory 2002 Available from: http://users.ox.ac.uk/ lina0174/vacuum.pd f.

4. Landau, L., A Course in Theoretical Physics: Quantum Mechanics. Vol. 3. 1982: Butterworth-Heinemann.

5. Landau, L., A Course in Theoretical Physics: Quantum Electrodynamics. Butterworth-Heinemann. Vol. 4. 1984.

6. Misner, C.T., K.; Wheeler, J., Gravitation. 21st ed. 1998: W. H. Freeman and Co.

7. Baez, J. What's the Energy Density of the Vacuum? 2011; Available from: http://math.ucr.edu/home/baez/vacuum.ht $\underline{\mathrm{ml}}$.
8. Landau, L., A Course in Theoretical Physics: The Classical Theory of Fields. Vol. 2. 1982: Butterworth-Heinemann.

9. Feynman Leighton Sands, The Feynman Lectures on Physics. Addison-Wesley, Reading, Massachusetts. Vol. 21989.

10. Landau Lifshitz, A Course in Theoretical Physics: Quantum Electrodynamics. Butterworth-Heinemann. Vol. 4. 1984. 